cancers. HER-2 is also important in cardiac development and in the response to cardiac injury. Trastusumab, as monotherapy or in combination with other cytotoxic agents, still produces a cardiotoxic effect in a minority of patients. Therefore, cardiac monitoring, with serial measurement of cardiac ejection fraction by echocardiography or multiple gated acquisition scans, is recommended. If there is significant decline in ejection fraction, trastusumab therapy should be suspended; if it has declined below normal, patients should be treated with angiotensin-converting enzyme inhibitors or other drugs to reduce afterload.

\section{Tyrosine kinase inhibitors}

A number of the new tyrosine kinase inhibitors are in development for the treatment of a wide range of cancers. Lapatinib, an inhibitor of both HER-1 and HER-2, appears to have a relatively safe cardiac toxicity profile. Other tyrosine kinase inhibitors that target a variety of oncogenic signalling pathways (often multiple) need to be monitored carefully as many induce prolongation of the QTc interval and have the potential to induce cardiac arrhythmia.

\section{References}

1 Lenz HJ. Management and preparedness for infusion and hypersensitivity reactions. Review. Oncologist 2007;12:601-9.

2 Hochberg J, Cairo MS. Tumour lysis syndrome: a current perspective. Haematologica 2008;93:9-13.

3 Warr DG. Chemotherapy- and cancerrelated nausea and vomiting. Curr Oncol 2008;15(Suppl 1):S4-9.

4 Yao X, Panichpisal K, Kurtzman N, Nugent K. Cisplatin nephrotoxicity: a review. Am J Med Sci 2007;334:115-24.

5 Sonis ST. A biological approach to mucositis. Review. J Support Oncol 2004;2: 21-32.

6 Chanan-Khan A, Srinivasan C, Czuczman MS. Prevention and management of cardiotoxicity from antineoplastic therapy. Review. J Support Oncol 2004;2:251-6.

\title{
Contemporary management of
}

\section{hepatocellular carcinoma}

Daniel Palmer BSc(Hons) MBChB MRCP PhD, Cancer Research UK Clinician Scientist and Honorary Consultant in Medical Oncology, Cancer Research UK Institute for Cancer Studies, University of Birmingham

\section{Clin Med 2008;8:442-7}

The liver is the organ most frequently involved in cancer, usually by metastases. Primary liver cancer (hepatocellular carcinoma (HCC)) is a major public health problem in developing countries, responsible for over 500,000 deaths per year. In the West its incidence is rising, in part due to the increasing prevalence of chronic hepatitis $\mathrm{C}$ virus (HCV) infection. ${ }^{1}$ A variety of treatment options exist but these are generally limited to carefully selected patients. Until recently there was no good systemic therapy, but an increased understanding of the molecular pathogenesis of HCC and the advent of novel targeted agents have seen the emergence of active therapies in this setting.

\section{Epidemiology and aetiology}

HCC is one of the commonest malignancies worldwide but with wide geographical variation (Table 1). It occurs almost exclusively on a background of chronic liver disease (Table 2), usually at the stage of cirrhosis. This has a significant impact on the mode of presentation, complicates diagnosis and limits therapeutic options. Knowledge of aetiological factors provides the opportunity for preventive strategies. A hepatitis B virus vaccination programme of inoculating neonates, initiated in Taiwan in the early 1980s, has resulted in a clear reduction in the incidence of childhood HCC. ${ }^{2}$ An effect on the incidence in adults may take a further 20 years to become apparent. There is also evidence to suggest that antiviral therapy in patients with chronic hepatitis $\mathrm{B}$ may reduce the incidence of HCC. ${ }^{3}$ There is no vaccine against $\mathrm{HCV}$, but antiviral drugs probably also reduce the incidence of HCC in that situation.

\section{Diagnosis}

The functional reserve of the liver is such that tumours can reach a considerable size before causing symptoms or signs, typically right upper quadrant pain, hepatomegaly and weight loss. Decompensation of chronic liver disease (variceal haemorrhage, ascites, encephalopathy) is also a frequent presentation. Less commonly, a tumour may rupture, resulting in severe abdominal

Table 1. Geographical incidence of hepatocellular carcinoma per 100,000 population.

\begin{tabular}{llcl} 
Incidence & Country & Men & Women \\
\hline \multirow{2}{*}{ Low } & UK & 1.6 & 0.8 \\
& USA (white) & 2 & 1 \\
& Australia & 1.1 & 0.5 \\
& Germany & 4 & 1.2 \\
& Denmark & 3.6 & 2.3 \\
Intermediate & Italy & 7.5 & 3.5 \\
& Spain & 7.5 & 4 \\
& Romania & 11.8 & 7.9 \\
& Argentina & 8 & 5 \\
& Japan & 20 & 5 \\
& Hong Kong & 32 & 7 \\
& Zimbabwe & 65 & 25 \\
& Senegal & 25 & 9 \\
& Taiwan & 85 & -
\end{tabular}


pain, shock and haemoperitoneum. Rarer presentations include hypoglycaemia, hypercalcaemia and polycythaemia due to tumour secretion of insulin-like growth factors, parathyroid-related hormones and erythropoietin, respectively.

HCC is increasingly diagnosed presymptomatically as a result of screening programmes. Computed tomography (CT) and magnetic resonance imaging (MRI) show enhancement in the arterial phase with relative hypovascularity ('wash-out') in the portal or late phases. The European Association for Study of the Liver (EASL) criteria state that in a patient with cirrhosis and a mass greater than $2 \mathrm{~cm}$ this radiological appearance, confirmed by two imaging modalities or combined with a serum alpha-fetoprotein (AFP) greater than $400 \mathrm{ng} / \mathrm{ml}$, is diagnostic of HCC without the need for histology. ${ }^{4}$ Although CT and MRI are currently the best imaging modalities, both techniques may miss up to $30 \%$ of lesions (as detected in the explanted liver following liver transplantation), especially those less than $1 \mathrm{~cm}$ in diameter.

Serum AFP is elevated in 70\% of patients. It is of diagnostic value in cirrhosis and has been used as a screening tool in high-risk populations. Although levels of $500 \mathrm{ng} / \mathrm{ml}$ or more can occur in benign liver diseases, a rising AFP is strongly suggestive of HCC. It may also be useful in monitoring the effects of treatment and for the detection of recurrence or progression following treatment.

For a liver mass not fulfilling the EASL criteria, a diagnosis of HCC requires histological confirmation. Fine-needle biopsy may be limited by sampling error, particularly for small lesions, and by difficulty in distinguishing well-differenti-

Table 2. Major risk factors for hepatocellular carcinoma.

- Chronic liver disease (usually at the stage of cirrhosis)

- Chronic hepatitis B virus infection

- Chronic hepatitis C virus infection

- Dietary exposure to aflatoxin

- Increasing age

- Male gender

\section{Key Points}

Diagnosis, treatment and prognosis of hepatocellular carcinoma are influenced by underlying chronic liver disease

Knowledge of risk factors provides scope for preventive strategies

Biopsy of a liver mass should be avoided before consultation with a hepatobiliary multidisciplinary team

Curative treatments (surgery, ablation) are applicable to only a minority of patients

Sorafenib is the first systemic agent to prolong survival in patients with advanced disease

KEY WORDS: chemo-embolisation, cirrhosis, hepatocellular carcinoma, liver transplantation, prevention, sorafenib

ated HCC from dysplasia or adenoma. Some groups believe that biopsy may risk tumour seeding along the needle track and recommend avoidance of the procedure in candidates for surgical resection or transplantation. Histological type is not of prognostic significance, with the exception of the fibrolamellar variant which typically occurs in younger patients without underlying chronic liver disease. In this setting, resection rates are higher and prognosis better (median survival five years), although this may reflect the younger age and absence of cirrhosis.

\section{Screening}

HCC predominantly occurs on the background of chronic liver disease so there is an opportunity to screen high-risk populations and some evidence that this can reduce disease-specific mortality. Current guidelines suggest that those in a high-risk group undergo six-monthly ultrasound examinations, serial AFP measurements appearing to be insufficiently sensitive. ${ }^{5}$

\section{Staging and prognosis}

Prognosis for most cancers is predominantly determined by tumour stage, but most HCCs occur on a background of cirrhosis which independently contributes to prognosis. Tumour size is a key prognostic factor. Vascular invasion increases with tumour size, but is an independent prognostic factor. Even large tumours can have a good prognosis following surgical resection in the absence of vascular invasion. Underlying cirrhosis may limit prognosis and can influence treatment options such as surgical resection which requires sufficient hepatic reserve to be performed safely. Prognostic models for HCC are therefore complex and should take into account tumour stage, degree of liver impairment, patient fitness and treatment efficacy. ${ }^{6}$

\section{Treatment}

\section{Liver resection}

Fewer than $20 \%$ of patients are suitable for resection, although this may increase as screening programmes detect tumours at an earlier stage. The ability to resect a tumour depends on its size, location, relation to blood vessels, and the underlying liver function. In patients without cirrhosis, up to $75 \%$ of the liver can be removed; hepatic resection is the treatment of choice for them, with five-year survival up to $70 \% .^{7}$ In cirrhotics, resection is associated with significant morbidity and mortality, although this has fallen below 5\% with improvements in surgical technique and postoperative care.

\section{Transplantation}

Liver transplantation has the potential to treat both tumour and underlying cirrhosis, although patients with viral hepatitis have a risk of reinfection in the new liver. The Milan criteria define a group of patients for whom transplantation can lead to survival similar to that of 
patients with benign end-stage liver disease (5-year survival 70\%). ${ }^{8}$ These criteria are used to select patients with HCC for transplant listing, although recent data indicate that the criteria for transplantation may be extended while still retaining good outcomes (Table 3)., ${ }^{8,9}$ Where available, transplantation is the treatment of choice for HCC in a cirrhotic liver.

A major limitation to transplantation is the supply of donor organs. This means a period of uncertain duration between listing and transplantation, with the risk that the tumour will grow beyond the criteria for transplant. Dropout, caused by disease progression to a level at which transplantation is no longer appropriate while awaiting a donor liver, may best be reduced by increasing the number/availability of donor organs. This may be helped by the use of living donors. Early data indicate that similar results are achieved to those with cadaveric organs if the Milan criteria are followed, with low mortality amongst donors. ${ }^{10}$

\section{Local ablation}

By many criteria, ablative therapies appear to be as effective as surgery in appropriately selected cases but, like surgery, are less effective once tumour size exceeds $5 \mathrm{~cm}$. In addition to increasing difficulty in achieving complete ablation, this probably reflects the increasing frequency of vascular invasion as tumours increase in size and, with it, the likelihood of metastasis.

Treatment is usually performed percu- taneously under image guidance. Several methods have been used, the most widely studied being percutaneous ethanol injection (PEI) and radiofrequency ablation (RFA). Injection of $90 \%$ ethanol under ultrasound guidance is technically straightforward, inexpensive, safe and, depending on the severity of underlying cirrhosis, can result in five-year survival of up to $50 \% .^{11}$

RFA is a localised thermal treatment producing tumour destruction by heating a probe inserted into the tumour to temperatures exceeding $60^{\circ} \mathrm{C}$. Randomised studies comparing RFA with PEI indicate that the former is associated with improved local control and likely improvement in survival. ${ }^{12}$

\section{Transarterial chemo-embolisation}

HCC is a highly vascularised tumour, mostly obtaining its blood supply via the hepatic artery, whereas normal liver parenchyma derives most of its from the portal vein. Transarterial chemo-embolisation (TACE) utilises selective catheterisation of the hepatic artery to deliver regional chemotherapy and embolise tumour-feeding arteries (Fig 1). Chemotherapy is injected, often mixed with lipiodol, an oily compound that accumulates preferentially in tumours, probably via enhanced permeability of leaky tumour vasculature and retention due to impaired lymphatic drainage. The aim is to retain the chemotherapy to increase tumour concentration and reduce systemic exposure. This is followed by embolisation of tumour-feeding arteries using one of a variety of embolic materials.

Table 3. Eligibility criteria for liver transplantation in patients with hepatocellular carcinoma.

Eligibility criteria

\begin{tabular}{ll}
\hline Milan & UCSF $^{9}$ \\
\hline One tumour $\leq 5 \mathrm{~cm}$ & One tumour $\leq 6.5 \mathrm{~cm}$ \\
or & or \\
Up to 3 nodules, each $\leq 3 \mathrm{~cm}$ & Up to 3 nodules, each $\leq 4.5 \mathrm{~cm}$ and \\
& total tumour diameter $\leq 8 \mathrm{~cm}$ \\
\hline
\end{tabular}

UCSF = University of California, San Francisco.

For chemo-embolisation to be performed safely, there must be adequate blood supply to the non-tumorous liver via the portal vein, so it is contraindicated in the presence of main portal vein thrombosis. Other contraindications include extrahepatic disease, advanced cirrhosis and poor performance status. Embolisation is frequently complicated by a characteristic syndrome of abdominal pain, fever and nausea, normally self-limiting but occasionally patients go on to develop liver abscess.

Randomised controlled trials (RCTs) and a meta-analysis have demonstrated a survival benefit for patients receiving TACE compared with supportive care. ${ }^{13}$ The general applicability of these data is limited by the relatively small sample size, the heterogeneity of the patient populations and the techniques used, with differences in the choice of chemotherapeutic and embolic agents and use of lipiodol.

\section{Radio-isotopes}

Radio-isotopes can also be delivered via the hepatic artery using yttrium-90labelled microspheres or iodine-131containing lipiodol. Iodine-131 lipiodol, a gamma emitter, can induce response rates of $40 \%$, while limiting radiation dose to normal liver to less than 20 Gy. Yttrium-90 is a beta emitter with shorter tissue penetration, which may further improve normal liver tolerance and reduce the risk of radiation hepatitis. ${ }^{14}$ Survival benefit has not yet been demonstrated.

A major limitation of loco-regional approaches is disease recurrence, which may represent the growth of pre-existing micrometastases from the primary tumour or the development of a new tumour on the background of cirrhosis. In the absence of an effective systemic agent, there are no conclusive data that adjuvant treatment can decrease the risk of tumour recurrence.

\section{Systemic therapies for hepatocellular carcinoma}

Most patients with HCC have multifocal disease, bilobar disease, extrahepatic disease and/or underlying cirrhosis. Surgery, 
ablation or chemo-embolisation is not indicated in such patients.

\section{Chemotherapy}

Objective radiological response rates for single-agent chemotherapy are low (10-15\%). The most widely used drug has been doxorubicin, although randomised trials have failed to discern a significant survival benefit. Combination chemotherapy can induce higher response rates (20-30\%) but with no significant impact on survival. ${ }^{15}$ Interpretation of the impact of chemotherapy is limited by the small size of clinical trials and the heterogeneous patient groups.

More recently, a large trial has investigated a novel thymidylate synthase inhibitor, nolatrexed, using doxorubicin as the comparator. Despite encouraging evidence of activity in earlier phase trials, the survival of patients receiving nolatrexed was significantly shorter than those in the control arm (6.9 months $v$ 4.7 months, $\mathrm{p}=0.0068$ ). The statistical assumptions used in the design of this trial were based on demonstrating superiority for nolatrexed but, since there were no obvious nolatrexed-related early deaths, it has been argued that this study provides evidence that doxorubicin may positively influence survival in appropriately selected patients. ${ }^{16}$

While conventional cytotoxic therapy has some activity against HCC, whether or not this translates into a survival advantage has still not been rigorously demonstrated.

Endocrine therapies. Endocrine therapies have been investigated in HCC based on reports of oestrogen receptor expression. Early small studies with tamoxifen showed promise but large prospective controlled studies have refuted any beneficial role. ${ }^{17}$

\section{Angiogenesis}

Development of a new blood supply is essential for a tumour to grow beyond the size at which oxygen enters by diffusion. Vascular endothelial growth factor (VEGF) is a key signalling protein stimu-
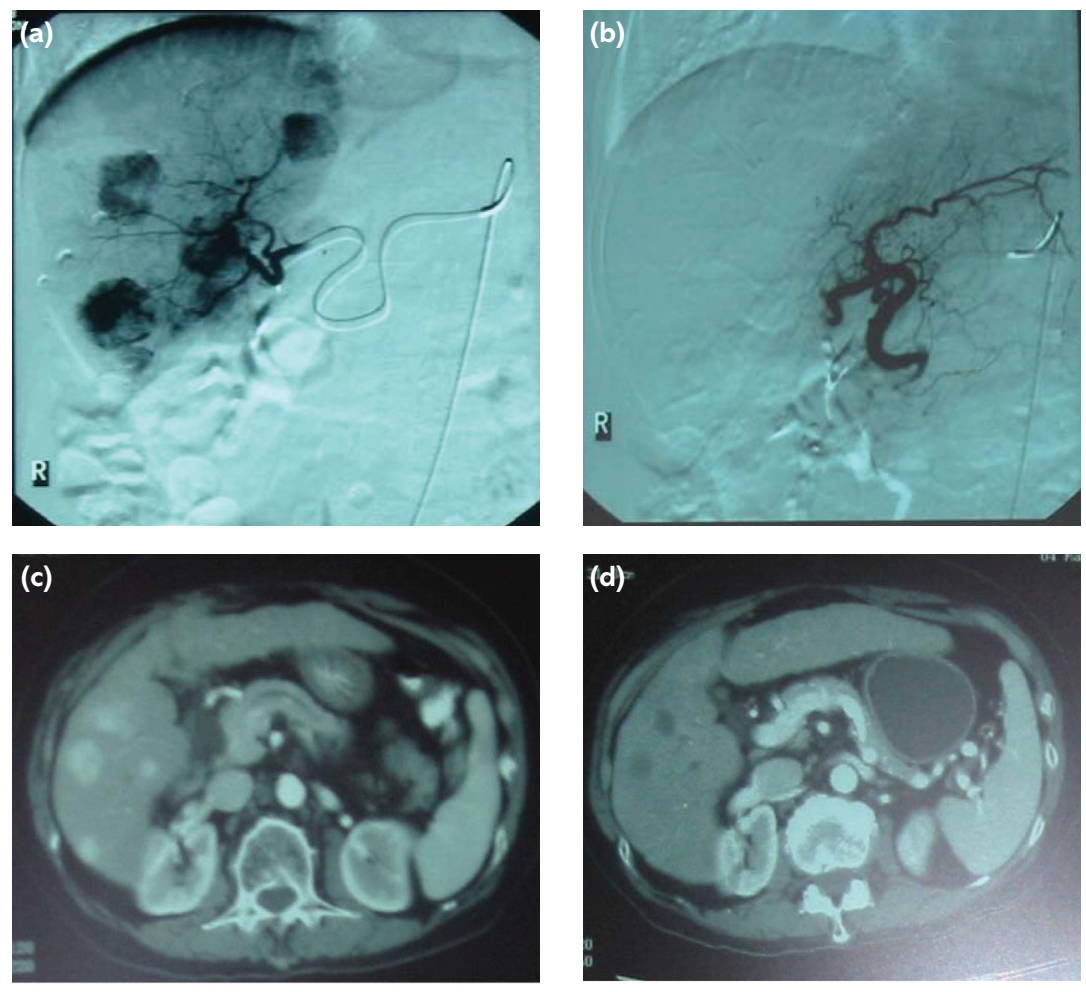

Fig1. Transarterial chemo-embolisation; (a) and (b) hepatic arterial angiography; (c) and (d) contrast-enhanced computed tomography. Pre-embolisation ((a) and (c)) demonstrate hypervascular multifocal hepatocellular carcinoma; post-embolisation ((b) and (d)) show that tumour circulation is abolished.

lated by hypoxia and acting predominantly on vascular endothelial cells via VEGF receptor-2 (VEGFR-2). This triggers a tyrosine kinase (TK) signalling cascade that promotes angiogenesis. This process can be inhibited pharmacologically either by small molecule inhibitors of VEGFR signalling (eg sorafenib) or through a monoclonal antibody targeted against VEGF preventing its interaction with receptor (eg bevacizumab) (Fig 2).

Sorafenib. Sorafenib is an oral multikinase inhibitor that targets both the raf/mek/erk growth factor signalling pathway which is over-expressed in $\mathrm{HCC}^{18}$ and also VEGFR TKs. It thus has direct antitumour and anti-angiogenic properties. The objective response rate to sorafenib was very low $(2.2 \%)$ in a phase 2 study, but a disease stability rate of $33 \%$ at four months and median survival of nine months were seen as sufficiently encouraging to proceed to a large RCT in which 602 patients were randomly assigned to either sorafenib or placebo. ${ }^{19}$ Treatment was well tolerated, with diar- rhoea, fatigue and skin toxicity common side effects. Sorafenib was associated with a significant improvement in overall survival compared with placebo (median survival 10.7 months $v 7.9$ months, $\mathrm{p}=0.00007) .^{20}$

Other novel agents. Although sorafenib is the first systemic agent to demonstrate a clear survival advantage in patients with HCC, other novel agents are showing promise in early phase trials. Sunitinib is another multikinase inhibitor targeting VEGF and platelet-derived growth factor receptor TKs. Phase 2 data are similar to sorafenib and phase 3 trials are planned. ${ }^{21}$ Bevacizumab is an anti-VEGF monoclonal antibody which, in combination with cytotoxic chemotherapy, is active in metastatic colorectal cancer. Phase 2 studies in patients with HCC have indicated modest activity. ${ }^{22}$

Tumour angiogenesis is regulated predominantly, but not exclusively, through VEGF signalling. The epidermal growth factor (EGF) and transforming growth factor-alpha play a major role in angio- 
genesis and in cancer cell proliferation and invasion. Both are active through the EGF receptor (EGFR) that is commonly overexpressed in HCC. Erlotinib is an orally active inhibitor of EGFR TK. Phase 2 studies have reported partial responses and encouraging disease stabilisation in HCC. ${ }^{23}$

\section{Combination therapy}

The complexity and redundancy within tumour cell and endothelial cell signalling suggest that inhibition of one single pathway is unlikely to meet with dramatic success. Increased understanding of these processes provides evidence for the rational combination of agents. For example, there is laboratory evidence of synergy between VEGF and EGF inhibition. The promising results for bevacizumab and erlotinib used singly have led to investigation of their use in combination, with encouraging results from a phase 2 study which reported a response rate of $22 \%$ and $55 \%$ progression-free survival, at 16 weeks. ${ }^{24} \mathrm{~A}$ phase 3 study comparing this combination with sorafenib is planned.

Pathways inhibited by novel targeted therapies, including raf/mek/erk, may also contribute to resistance to conventional chemotherapeutic agents. Combination of these novel agents with chemotherapy may overcome chemoresistance. A randomised phase 2 study has addressed this question by combining sorafenib with systemic doxorubicin. Combination therapy was well tolerated and associated with a significant prolongation of overall survival compared with doxorubicin alone (13.7 months $v 6.5$

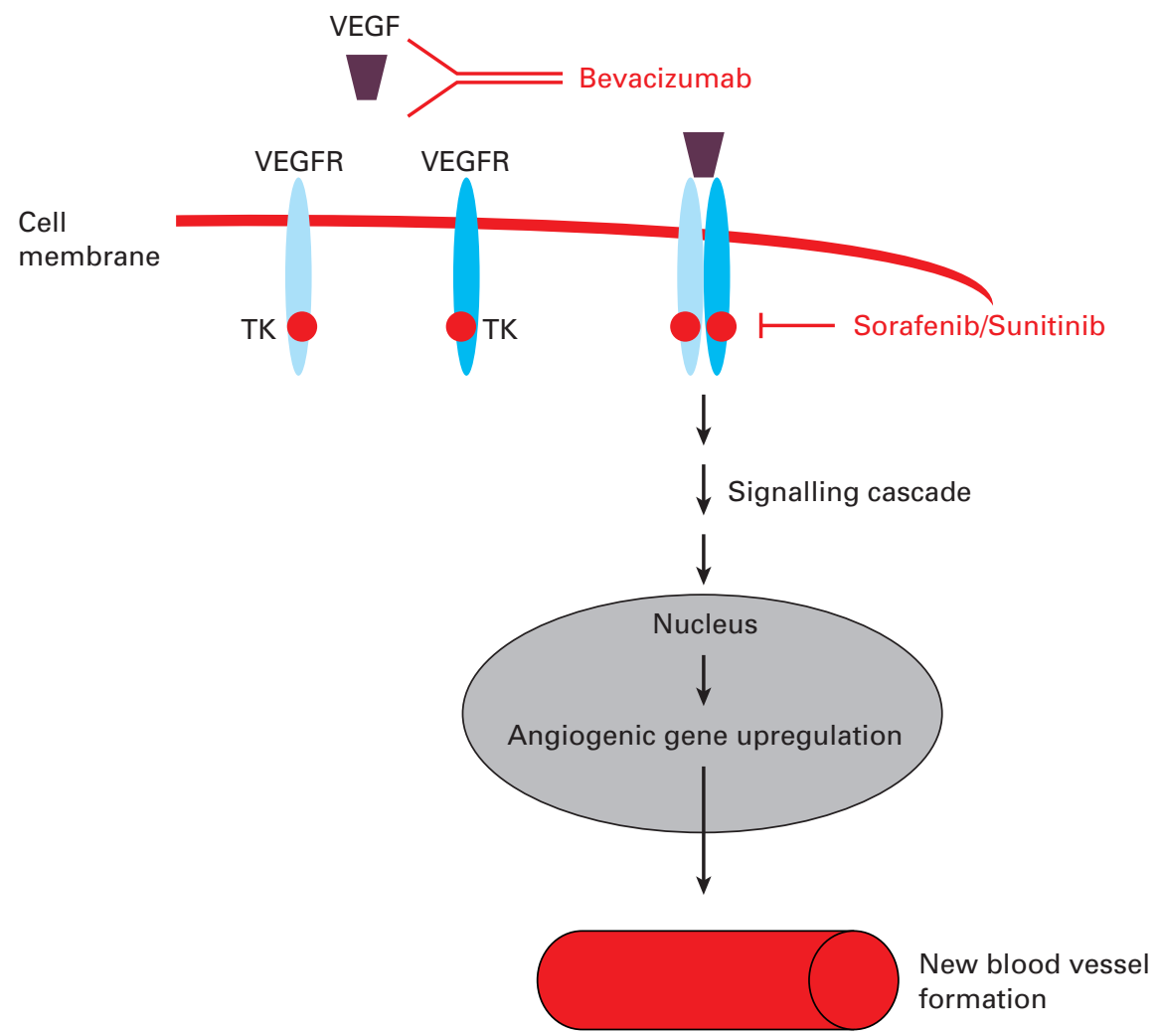

Fig 2. Vascular endothelial growth factor (VEGF) signalling and targets for antiangiogenic therapeutics. Hypoxia stimulates the production of VEGF, which engages its receptor (VEGFR) on the surface of vascular endothelial cells. Oligomerisation of receptors promotes tyrosine kinase (TK) activity of the intracellular domain of the receptor. This in turn activates a signalling cascade, culminating in the upregulation of genes encoding proteins that effect new blood vessel formation. This pathway can be inhibited at the level of VEGF ligand (eg by bevacizumab) or by inhibition of receptor TK activity (eg by sorafenib or sunitinib).

months, $\mathrm{p}=0.049) .^{25} \mathrm{~A}$ further randomised trial of the combination using sorafenib as the control arm is required to establish whether the benefit is attributable to synergy between the two agents or to sorafenib alone.

\section{Conclusions}

HCC poses a significant health burden worldwide. Identification of high-risk groups and advances in diagnostic radiology provide scope for earlier diagnosis and treatment. Significant progress is being made in the prevention of hepatitis B-related HCC, but hepatitis Crelated HCC is increasing in the West and therapeutic advances in established disease have been modest.

Transplantation, resection and ablative therapies are effective in patients with small tumours but are applicable to only a minority. Chemo-embolisation may offer palliation in a subset of patients with preserved liver function and patent portal vein.

Sorafenib is the first active systemic agent able to prolong survival for patients with advanced HCC, heralding an era of other novel agents and combinations. The advent of active systemic therapies for HCC also creates the opportunity for their use as an adjuvant to surgery and other locoregional therapies, with the aim of decreasing recurrence/progression and as a bridge to liver transplantation where prevention of disease progression may be a valuable outcome.

A multitude of agents and combinations are becoming available and decisions have to be made about which agents should progress to large-scale phase 3 trials. Traditionally, radiological response has been the preferred endpoint for phase 2 trials for conventional chemotherapy. However, many newer agents may not cause tumour shrinkage but rather disease stabilisation. This will require novel trial design incorporating, for example, time to progression or progression-free survival end-points, necessitating serial radiological assessments.

A critical challenge in the development of these therapies is identifying the presence of the relevant target in the tumour 
and to correlate this with response so that patients most likely to benefit can be selected.

\section{References}

1 El-Serag HB, Mason AC. Rising incidence of hepatocellular carcinoma in the United States. N Engl J Med 1999;340:745-50.

2 Chang MH, Chen CJ, Lai MS et al. Universal hepatitis B vaccination in Taiwan and the incidence of hepatocellular carcinoma in children. Taiwan Childhood Hepatoma Study Group. N Engl J Med 1997;336:1855-9.

3 Liaw YF, Sung JJ, Chow WC et al. Lamivudine for patients with chronic hepatitis B and advanced liver disease. N Engl J Med 2004;351:1521-31.

4 Bruix J, Sherman M, Llovet JM et al; EASL Panel of Experts on HCC. Clinical management of hepatocellular carcinoma. Conclusions of the Barcelona-2000 EASL conference. European Association for the Study of the Liver. J Hepatol 2001;35:421-30.

5 Bruix J, Sherman M; Practice Guidelines Committee, American Association for the Study of Liver Diseases. Management of hepatocellular carcinoma. Hepatology 2005; 42:1208-36.

6 Palmer DH, Johnson PJ. Prognostic Factors in Hepatocellular Carcinoma. In: UICC Prognostic Factors in Cancer, 3rd edn. New Jersey: Wiley and Sons, 2006.

7 Vauthey JN, Klimstra D, Franceschi D et al. Factors affecting long-term outcome after hepatic resection for hepatocellular carcinoma. Am J Surg 1995;169:28-34.

8 Mazzaferro V, Regalia E, Doci R et al. Liver transplantation for the treatment of small hepatocellular carcinomas in patients with cirrhosis. N Engl J Med 1996;334:693-9.

9 Yao FY, Ferrell L, Bass NM et al. Liver transplantation for hepatocellular carcinoma: expansion of the tumor size limits does not adversely impact survival. Hepatology 2001;33:1394-403.
10 Gondolesi GE, Roayaie S, Munoz L et al. Adult living donor liver transplantation for patients with hepatocellular carcinoma: extending UNOS priority criteria. Ann Surg 2004;239:142-9.

11 Livraghi T, Giorgio A, Marin G et al. Hepatocellular carcinoma and cirrhosis in 746 patients: long-term results of percutaneous ethanol injection. Radiology 1995; 197:101-8.

12 Shiina S, Teratani T, Obi S et al. A randomized controlled trial of radiofrequency ablation with ethanol injection for small hepatocellular carcinoma. Gastroenterology 2005;129:122-30.

13 Bruix J, Sala M, Llovet JM. Chemoembolization for hepatocellular carcinoma. Review. Gastroenterology 2004;127 (5 Suppl 1):S179-88.

14 Lau WY, Ho S, Leung TW et al. Selective internal radiation therapy for nonresectable hepatocellular carcinoma with intraarterial infusion of 90yttrium microspheres. Int J Radiat Oncol Biol Phys 1998;40:583-92.

15 Palmer DH, Hussain SA, Johnson PJ. Systemic therapies for hepatocellular carcinoma. Review. Expert Opin Investig Drugs 2004;13:1555-68.

16 Gish RG, Porta C, Lazar L et al. Phase III randomized controlled trial comparing the survival of patients with unresectable hepatocellular carcinoma treated with nolatrexed or doxorubicin. J Clin Oncol 2007;25:3069-75.

17 Tamoxifen in treatment of hepatocellular carcinoma: a randomised controlled trial. CLIP Group (Cancer of the Liver Italian Programme). Lancet 1998;352:17-20.

18 Ito $\mathrm{Y}$, Sasaki $\mathrm{Y}$, Horimoto $\mathrm{M}$ et al. Activation of mitogen-activated protein kinases/extracellular signal-regulated kinases in human hepatocellular carcinoma. Hepatology 1998;27:95-18.

19 Abou-Alfa GK, Schwartz L, Ricci S et al. Phase II study of sorafenib in patients with advanced hepatocellular carcinoma. J Clin Oncol 2006;24:4293-300.
20 Llovet J, Ricco S, Mazzaferro V et al; for the SHARP Investigators Study Group. Sorafenib improves survival in advanced Hepatocellular Carcinoma (HCC): Results of a Phase III randomized placebocontrolled trial (SHARP trial). J Clin Oncol 2007 ASCO Annual Meeting Proceedings Part I, vol 25, No. 18S (June 20 Supplement), 2007: abstract LBA1.

21 Zhu AX, Sahani DV, di Tomaso E et al. A phase II study of sunitinib in patients with advanced hepatocellular carcinoma. J Clin Oncol 2007 ASCO Annual Meeting Proceedings Part I, vol 25, No. 18S (June 20 Supplement), 2007:abstract 4637.

22 Schwartz JD, Schwartz M, Lehrer D et al. Bevacizumab in hepatocellular carcinoma (HCC) in patients without metastases and without invasion of the portal vein. J Clin Oncol 23:338s, 2005 suppl:abstr 4122.

23 Philip PA, Mahoney MR, Allmer C et al. Phase II study of Erlotinib (OSI-774) in patients with advanced hepatocellular cancer. J Clin Oncol 2005;23:6657-63.

24 Thomas MB, Chadha R, Iwasaki M, Glover $\mathrm{K}$, Abbruzzese JL. The combination of bevacizumab (B) and erlotinib (E) shows significant biological activity in patients with advanced hepatocellular carcinoma (HCC). J Clin Oncol 2007 ASCO Annual Meeting Proceedings Part I, vol 25, No. 18S (June 20 Supplement), 2007:abstract 4567.

25 Abou-Alfa G, Johnson P, Knox J et al. Preliminary results from a Phase II, randomized, double-blind study of sorafenib plus doxorubicin versus placebo plus doxorubicin in patients with advanced hepatocellular carcinoma. Proceedings of the 2007 meeting of the European Cancer Organisation. Eur J Cancer 2007;5(Suppl): 259, abstract 3500 . 